\title{
A New On-line Exponential Parameter Estimator without Persistent Excitation
}

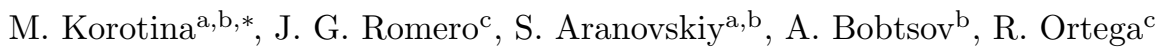 \\ ${ }^{a}$ IETR - CentaleSupélec, Avenue de la Boulaie, 35576 Cesson-Sévigné, France \\ ${ }^{b}$ Faculty of Control Systems and Robotics, ITMO University, 197101 Saint-Petersburg, Russia \\ ${ }^{c}$ Departamento Académico de Sistemas Digitales, ITAM, Río hondo 1, Progreso Tizapán, 01080, Mexico City, Mexico
}

\begin{abstract}
In this paper we propose a new algorithm that estimates on-line the parameters of a classical vector linear regression equation $\mathbf{Y}=\Omega \theta$, where $\mathbf{Y} \in \mathbb{R}^{n}, \Omega \in \mathbb{R}^{n \times q}$ are bounded, measurable signals and $\theta \in \mathbb{R}^{q}$ is a constant vector of unknown parameters, even when the regressor $\Omega$ is not persistently exciting. Moreover, the convergence of the new parameter estimator is global and exponential and is given for both, continuous-time and discrete-time implementations. As an illustration example we consider the problem of parameter estimation of a linear timeinvariant system, when the input signal is not sufficiently exciting, which is known to be a necessary and sufficient condition for the solution of the problem with standard gradient or least-squares adaptation algorithms.
\end{abstract}

Keywords: Parameter estimation, Persistent excitation, Interval excitation, Dynamic regressor extending and mixing, Nonlinear filter

\section{Introduction and Problem Formulation}

One of the central problems in control and systems theory, that has attracted the attention of many researchers for several years, is the estimation of the parameters that appear in the mathematical model that describes the systems behavior, usually a differential or a difference equation. A typical paradigm, which appears in system identification 12], adaptive control [21], filtering and prediction [8, reinforcement learning [11, and in many other application areas, is when the unknown parameters and the measured data are linearly related in a so-called linear regression equation (LRE). Classical solutions for this problem are gradient and least-squares estimators. The main drawback of these schemes is that convergence of the parameter estimates relies on the availability of signal excitation, a feature that is codified in the restrictive assumption of persistency of excitation (PE) of the regressor vector. Moreover, their transient performance is highly unpredictable and only a weak monotonicity property of the estimation errors can be guaranteed.

In recent years, various efforts to ease the PE requirement have been suggested, such as concurrent [6], or composite learning [20] that, in the spirit of off-line estimators, incorporate the monitoring of past data to build a stack of suitable regressor vectors. Another approach that has been extensively studied by the authors is the dynamic regressor extension and mixing (DREM) parameter estimation procedure, which was first proposed in [2] for continuous-time (CT) and in [4] for discrete-time (DT) systems. The construction of DREM estimators proceeds

\footnotetext{
${ }^{*}$ Corresponding author

Email address: marina.korotina@centralesupelec.fr (M. Korotina)

This work was supported by theRussian Science Foundation, project no. 18-19-00627, https://rscf.ru/project/18-19-00627/.
} 
in two steps, first, the inclusion of a free, stable, linear operator that creates an extended matrix LRE. Second, a nonlinear manipulation of the data that allows to generate, out of an $q$-dimensional LRE, $q$ scalar, and independent, LREs. DREM estimators have been successfully applied in a variety of identification and adaptive control problems, both, theoretical and practical ones, see [16, 17] for an account of some of these results.

A very important feature of the new concurrent and composite learning estimators is that parameter convergence is guaranteed under the extremely weak assumption of interval excitation (IE) [9 - the interested reader is refered to [16] where a detailed discussion of these methods, and the connections of them with existing results, is thoroughly discussed. This key property was also recently established for a version of DREM reported in [7, that has the additional feature of ensuring convergence in finite-time - see also [17, Propositions 6 and 7]. A potential drawback of this DREM algorithm is that it relies on fixing the initial conditions of some filters, which may adversely affect the robustness of the estimator, [17, Remark 7] and [19].

In the recent paper [5] a procedure to generate, from a scalar LRE, new scalar LREs where the new regressor satisfies some excitation conditions, even in the case when the original regressor is not exciting, was proposed. Instrumental for the development of the new adaptation algorithm is to borrow the key idea of the parameter estimation based observer proposed in [14, later generalized in [15, to generate the new LRE that includes some free signals. Then, applying the energy pumping-and-damping injection principle of [23], we select these signals to guarantee some excitation properties of the new regressor. Unfortunately, to prove that the aforementioned excitation properties guarantee parameter convergence it is necessary to assume some a priori non-verifiable conditions $[5$, behavior of some of the components of the trajectories of the estimator.

In this paper we extend the DREM procedure and, in particular the results of [5], in several directions with our main contributions summarized as follows.

C1 We give a definite answer to the question of ensuring that the new regressor is PE assuming only the extremely weak condition of IE of the original vector regressor. Towards this end, still abiding to the energy pumpingand-damping injection principle of [23], we propose a new selection of the free signals of the LRE generator of [5] for which the exponential convergence proof can be completed without any additional assumptions. In summary, the main contribution of the paper is to construct a new LRE with a regressor which is PE, even though the original regressor $\Omega$ is not.

C2 We illustrate our result with the important example of parameter identification of linear time-invariant (LTI) systems. It is well-known that a necessary and sufficient condition for global exponential convergence of the standard gradient (or least squares) estimators is the sufficient richness condition of the plants input signal [21, Theorems 2.7.2 and 2.7.3], which is equivalent to the $\mathrm{PE}$ of the original regressor. We prove here that this condition is not necessary, and show that it is possible to exponentially estimate the parameters of the plant under the very weak assumption of IE of the original regressor.

C3 Motivated by the practical relevance of DT implementations we extend the LRE generator procedure of [5], which was given for the CT case, to the DT case. Also, we propose the new DT signals that yield essentially the same results of $\mathrm{CT}$ mentioned in $\mathbf{C} 1$ and $\mathbf{C 2}$ above. 
The remainder of the paper is organized as follows. Some background material of the Kreisselmeier regressor erator procedure of [5] is given in Section 2. In Section 3 we present our main result discussed in C1 above. In Section 4 we briefly discuss the results. Section 5 presents the application to the parameter identification of LTI systems mentioned in C2. Simulation results of the DT version of the result are presented in Section 6. The paper is wrapped-up with concluding remarks and future research in Section 7 . To simplify the reading, a list of acronyms

Notation. $I_{n}$ is the $n \times n$ identity matrix. $\mathbb{Z}_{>0}$ and $\mathbb{Z}_{\geq 0}$ denote the positive and non-negative integer numbers, respectively. For $x \in \mathbb{R}^{n}$, we denote the Euclidean norm $|x|^{2}:=x^{\top} x$. CT signals $s: \mathbb{R}_{\geq 0} \rightarrow \mathbb{R}$ are denoted $s(t)$, while for DT sequences $s: \mathbb{Z}_{\geq 0} \rightarrow \mathbb{R}$ we use $s(k):=s\left(k T_{s}\right)$, with $T_{s} \in \mathbb{R}_{>0}$ the sampling time. The action of an a sequence $s(k)$ we use $\mathcal{H}[s](k)$. In particular, we define the derivative operator $p^{n}[s](t)=$ : $\frac{d^{n} s(t)}{d t^{n}}$ and the delay operator $q^{ \pm n}[s](k)=: s(k \pm n)$, where $n \in \mathbb{Z}_{>0}$. When a formula is applicable to CT signals and DT sequences the time argument is omitted.

\section{Background Material}

In this section we present the following preliminary results which are instrumental for the development of our new results.

- Derivation and properties of the KRE with the DREM estimator in CT [16, Proposition 3] [2, Proposition 1] and in DT [18, Proposition 3].

- Generation of new LREs for CT [5, Proposition 12] and DT. Since the derivation of the DT LREs is reported here for the first time, we present also a detailed proof of the proposition.

- Properties of the standard gradient estimator for the new LRE in CT [2, Proposition 1] and in DT [18, Proposition 3].

The following definitions will be used in the sequel.

Definition 1. A bounded signal $s \in \mathbb{R}^{r \times s}$ is PE [21] if

$$
\int_{t}^{t+T_{a}} s(\tau) s^{\top}(\tau) d \tau \geq C_{a} I_{r}
$$

for some $C_{a}>0$ and $T_{a}>0$ and for all $t \geq 0$ in $\mathrm{CT}$ and

$$
\sum_{j=k}^{k+k_{b}} s(j) s^{\top}(j) \geq C_{b} I_{r},
$$

\footnotetext{
${ }^{2}$ As explained in Section 4 there is a slight modification of the $z(t)$ dynamics with respect to the one given in [5, Proposition 1], namely the addition of a signal $u_{4}(t)$, that is introduced to simplify the proof of boundedness of $z(t)$.
} 
for some $C_{b}>0$ and $k_{b} \in \mathbb{Z}_{>0}$ and for all $k \in \mathbb{Z}_{\geq 0}$ in DT.

It is said to be IE [9, 10] if

$$
\int_{0}^{t_{c}} s(\tau) s^{\top}(\tau) d \tau \geq C_{c} I_{r}
$$

for some $C_{c}>0$ and $t_{c}>0$ in $\mathrm{CT}$ and

$$
\sum_{j=0}^{k_{d}} s(j) s^{\top}(j) \geq C_{d} I_{r}
$$

80 for some $C_{d}>0$ and $k_{d} \in \mathbb{Z}_{>0}$ in DT.

Proposition 1 (Construction of the KRE). Consider the LRE

$$
\mathbf{Y}=\Omega \theta
$$

where $\mathbf{Y} \in \mathbb{R}^{n}, \Omega \in \mathbb{R}^{n \times q}$ are bounded, measurable signals and $\theta \in \mathbb{R}^{q}$ is a constant vector of unknown parameters. Fix the constants $\lambda>0, g>0,0<\alpha<1$, and define the signals

$$
\begin{aligned}
Z & =\mathcal{H}\left[\Omega^{\top} \mathbf{Y}\right] \\
\Psi & =\mathcal{H}\left[\Omega^{\top} \Omega\right] \\
\mathcal{Y} & =\operatorname{adj}\{\Psi\} Z \\
\Delta & =\operatorname{det}\{\Psi\},
\end{aligned}
$$

where

$$
\mathcal{H}[s]=\left\{\begin{array}{lll}
\frac{g}{p+\lambda}[s](t) & \text { in } & C T \\
\frac{g}{q-\alpha}[s](k) & \text { in } D T,
\end{array}\right.
$$

and $\operatorname{adj}\{\cdot\}$ denotes the adjugate matrix ${ }^{3}$

P1 The signal $\Delta$ verifies

$$
\Delta \geq 0
$$

P2 The following implications are true [3, Proposition 1]

$$
\Omega \text { is }\left\{\begin{array} { c } 
{ \mathrm { IE } } \\
{ \mathrm { PE } }
\end{array} \Rightarrow \Delta \text { is } \left\{\begin{array}{c}
\mathrm{IE} \\
\mathrm{PE}
\end{array}\right.\right.
$$

P3 The $q$ scalar LREs

$$
\mathcal{Y}_{i}=\Delta \theta_{i}, i \in\{1,2, \ldots, q\},
$$

hold.

\footnotetext{
${ }^{3}$ Notice that a state space realization of the operator $\mathcal{H}: s \mapsto y$ is $\dot{y}(t)=-\lambda y(t)+g s(t)$ in CT and $y(k+1)=\alpha y(k)+g s(k)$ in DT.
} 
Proof. A detailed proof of the proposition may be found in [5]. For the sake of completeness we give below a brief sketch of it.

From (1), the extended LRE $\Omega^{\top} \mathbf{Y}=\Omega^{\top} \Omega \theta$ follows. Then, due to the linearity and BIBO stability of the chosen $\mathcal{H}[s]$, for the signals $Z$ and $\Psi$ it holds $Z=\Psi \theta$. Recalling that the identity $\operatorname{adj}\{\Psi\} \Psi=\operatorname{det}\{\Psi\} I$, which holds even for a singular $\Psi$, scalar LREs (5) follow.

The matrix $\Psi$ is generated applying $\mathcal{H}$ to the semi-positive-definite matrix $\Omega^{\top} \Omega$, and it is straightforward to show that $\Psi$ is also a semi-positive-definite matrix; thus, (3) follows.

Finally, the implication (4) can be established by solving the differential or difference equation defined by $\mathcal{H}$ in CT or DT, respectively, and deriving a lower bound for the eigenvalues of $\Psi$; see [3, 5] for details.

Proposition 2 (Generation of new LREs). Consider the scalar LREs 54 . Define the dynamic extension via the following (difference and differential) equations

$$
\begin{aligned}
\mathfrak{d}[z] & =u_{2} \mathcal{Y}+u_{3} z+u_{4}, z(0)=0 \\
\mathfrak{d}[\xi] & =A \xi+b, \xi(0)=\operatorname{col}(0,0) \\
\mathfrak{d}[\Phi] & =A \Phi, \Phi(0)=\operatorname{col}(1,0)
\end{aligned}
$$

where the operator $\mathfrak{d}[\cdot]$ is defined as

$$
\mathfrak{d}[u]=\left\{\begin{array}{lll}
p[u](t) & \text { in } & C T \\
q[u](k) & \text { in } & D T
\end{array}\right.
$$

and we defined

$$
A:=\left[\begin{array}{ll}
A_{11} & u_{1} \\
u_{2} \Delta & u_{3}
\end{array}\right], b:=\left[\begin{array}{c}
-u_{1} z \\
u_{4}
\end{array}\right],
$$

with $u_{i} \in \mathbb{R}, i=1, \ldots, 4$, arbitrary signals and

$$
A_{11}=\left\{\begin{array}{lll}
0 & \text { in } & C T \\
1 & \text { in } & D T
\end{array}\right.
$$

The new LRE

$$
Y=\Phi_{2} \theta
$$

holds with

$$
Y:=z-\xi_{2},
$$

and $\Phi_{2}$ the second component of the vector $\Phi$ defined in $6 \mathrm{c}$.

Proof. [DT version 5 Notice that, since $\theta$ is constant, we can write

$$
\theta(k+1)=\theta(k)+u_{1}(k)[z(k)-z(k)], \theta(0)=\theta
$$

\footnotetext{
${ }^{4}$ To simplify the notation we omit the subindex $i$ in the proposition.

${ }^{5}$ The proof of the CT case may be found in [5].
} 
Combining (6a) and (11), and using (5), we can write the "virtual" LTV system

$$
x(k+1)=A(k) x(k)+b(k),
$$

with $x(k):=\operatorname{col}(\theta(k), z(k)), A(k)$ and $b(k)$ defined in $(8)$ and initial conditions

$$
x(0)=\left[\begin{array}{l}
\theta \\
0
\end{array}\right] .
$$

Define the error signal

$$
e(k):=\xi(k)-x(k),
$$

which satisfies $e(k+1)=A(k) e(k)$. Consequently, from (14) and the properties of the signals $\Phi(k)$ defined in 6c), we get

$$
\begin{aligned}
x(k) & =\xi(k)-\Phi(k) e_{1}(0) \\
& =\xi(k)+\Phi(k) \theta
\end{aligned}
$$

where, to get the second identity, we took into account 13 and the initial conditions in $6 \mathrm{~b}$.

Now

$$
\left[\begin{array}{l}
\mathcal{Y}(k) \\
z(k)
\end{array}\right]=\left[\begin{array}{cc}
\Delta(k) & 0 \\
0 & 1
\end{array}\right] x(k)=\left[\begin{array}{cc}
\Delta(k) & 0 \\
0 & 1
\end{array}\right](\xi(k)+\Phi(k) \theta) .
$$

The proof is completed rewriting the latter as

$$
\left[\begin{array}{c}
\mathcal{Y}(k) \\
z(k)
\end{array}\right]-\left[\begin{array}{c}
\Delta(k) \xi_{1}(k) \\
\xi_{2}(k)
\end{array}\right]=\left[\begin{array}{c}
\Delta(k) \Phi_{1} \\
\Phi_{2}
\end{array}\right] \theta
$$

where 10 corresponds to the second row of this matrix equation.

Proposition 3 (Convergence properties of the gradient estimator). Consider the scalar LRE (10) of Proposition 2 with the gradient estimator

$$
\dot{\hat{\theta}}(t)=\gamma \Phi_{2}(t)\left[Y(t)-\Phi_{2}(t) \hat{\theta}(t)\right]
$$

in $\mathrm{CT}$ and

$$
\hat{\theta}(k+1)=\hat{\theta}(k)+\frac{\gamma \Phi_{2}(k)}{1+\gamma \Phi_{2}^{2}(k)}\left[Y(k)-\Phi_{2}(k) \hat{\theta}(k)\right] .
$$

in DT with $\gamma>0$.

The following equivalences are true:

$$
\begin{aligned}
\hat{\theta} \rightarrow \theta \Leftrightarrow \Phi_{2} \notin\left\{\begin{array}{ccc}
\mathcal{L}_{2} & \text { in } & C T \\
\ell_{2} & \text { in } & D T
\end{array}\right. \\
\hat{\theta} \rightarrow \theta,(\exp ) \Leftrightarrow \Phi_{2} \in \mathrm{PE} .
\end{aligned}
$$

Proof. A detailed proof of the proposition may be found in [2, Proposition 1] for CT and in [18, Proposition 3] for DT. For the sake of completeness we give below a brief sketch of it. 
Consider the estimation error $\tilde{\theta}:=\hat{\theta}-\theta$. In $\mathrm{CT}$, the parameter error equation takes the form

$$
\dot{\tilde{\theta}}(t)=-\gamma \Phi_{2}^{2}(t) \tilde{\theta}(t)
$$

and in DT is

$$
\tilde{\theta}(k+1)=\frac{1}{1+\gamma \Phi_{2}^{2}(k)} \tilde{\theta}(k) .
$$

For both error dynamics equations, proofs of the implications of Proposition 3 are widely known in adaptive control [8, 21].

\section{Main Result}

In this section we give the main result of the paper, namely the selection of the signals $u_{i}, i=1, \ldots, 4$, in (6)-(8) that ensure the new regressor $\Phi_{2}$ is PE under the very weak assumption of IE of the regressor $\Omega$ of the original LRE (1). In the DT case, it is necessary to impose an additional assumption on a tuning parameter.

Proposition 4. Consider the LRE (1) and the KRE construction of Proposition 1 . Assume $\Omega$ is IE. Consider the dynamics (6)-(8) with the signals

$$
u(t)=\left[\begin{array}{c}
u_{1}(t) \\
u_{2}(t) \\
u_{3}(t) \\
u_{4}(t)
\end{array}\right]=\left[\begin{array}{c}
-\mu \Delta(t) \Phi_{1}(t) \\
\mu \Phi_{1}(t) \\
-\tilde{V}(t) \\
{[\tilde{V}(t)-\mu] z(t)}
\end{array}\right]
$$

in $\mathrm{CT}$ and

$$
u(k)=\left[\begin{array}{c}
u_{1}(k) \\
u_{2}(k) \\
u_{3}(k) \\
u_{4}(k)
\end{array}\right]=\left[\begin{array}{c}
-T \mu \Delta(k) \Phi_{1}(k) \\
T \mu \Phi_{1}(k) \\
1-T \tilde{V}(k) \\
{[T \tilde{V}(k)-b] z(k)}
\end{array}\right]
$$

in DT, where

$$
\tilde{V}:=\frac{1}{2}\left(\Phi_{1}^{2}+\Phi_{2}^{2}\right)-\beta,
$$

with $0<b<1, \beta>\frac{1}{2}, \mu>0$ and $T>0$ is a small number such that we can assum $\underbrace{6}$

$$
T^{2} \approx 0
$$

F1 The signals $z, \xi$ and $\Phi$ are bounded.

F2 $\Phi_{2}$ is PE.

Proof. The proof proceeds in the following three steps.

- Proof of boundedness of $\Phi$.

- Proof of PE of $\Phi_{2}$.

\footnotetext{
${ }^{6}$ See the second bullet in Section 4 for the motivation to include this assumption.
} 
- Proof of boundedness of $z$ and $\xi$.

Although the arguments for the CT and the DT case are similar, for the sake of clarity, we present them in separate subsections whenever needed.

(i) Proof of boundedness of $\Phi(t)$

Replacing (18) in (6)-8) yields

$$
\begin{aligned}
& \dot{\Phi}_{1}(t)=-\mu \Delta(t) \Phi_{2}(t) \Phi_{1}(t), \\
& \dot{\Phi}_{2}(t)=\mu \Delta(t) \Phi_{1}^{2}(t)-\tilde{V}(t) \Phi_{2}(t) .
\end{aligned}
$$

From 20 and the equations of $\Phi(t)$ above we immediately get

$$
\dot{\tilde{V}}(t)=-\Phi_{2}^{2}(t) \tilde{V}(t)
$$

from which we conclude the invariance of the set

$$
\Omega:=\left\{\Phi \in \mathbb{R}^{2} \mid \frac{1}{2}\left(\Phi_{1}^{2}+\Phi_{2}^{2}\right)=\beta\right\} .
$$

Now, invoking the initial condition constraint $\Phi_{1}(0)=1$, and $\Phi_{2}(0)=0$, we have

$$
\begin{aligned}
& \frac{1}{2}\left(\Phi_{1}^{2}(0)+\Phi_{2}^{2}(0)\right)=\frac{1}{2} \\
& \Leftrightarrow \tilde{V}(0)+\beta=\frac{1}{2} \\
& \Leftrightarrow \tilde{V}(0)=\frac{1}{2}-\beta \\
& \Rightarrow \tilde{V}(0)<0,
\end{aligned}
$$

where we used $\beta>\frac{1}{2}$ to get the last implication. The latter inequality implies that the trajectory starts inside the disk delimited by the set $\Omega$. This, together with the invariance of the set implies, that the whole trajectory $\left(\Phi_{1}(t), \Phi_{2}(t)\right)$ is inside this disk, that is,

$$
\tilde{V}(t) \leq 0, \forall t \geq 0
$$

Replacing the bound above in 23 we have that $\dot{\tilde{V}}(t) \geq 0$ from which we conclude that $\tilde{V}(t)$ is non-decreasing, hence

$$
\tilde{V}(t) \geq \tilde{V}(0)=\frac{1}{2}-\beta
$$

Combining the bounds 25 and 26 we conclude that

$$
1 \leq \Phi_{1}^{2}(t)+\Phi_{2}^{2}(t) \leq 2 \beta
$$

The bounds above can be further sharpened as follows. From the constant $\mu(t) \geq 0,22 \mathrm{~b}),(25)$, (3), and recalling the initial condition $\Phi_{2}(0)=0$, it follows that $\dot{\Phi}_{2}(t) \geq 0$ and, consequently, $\Phi_{2}(t) \geq 0$ for all $t$. Moreover,

$$
\dot{\Phi}_{1}(t)=-\mu \Delta(t) \Phi_{2}(t) \Phi_{1} \leq 0
$$

hence $\Phi_{1}(t)$ is not increasing and, recalling that $\Phi_{1}(0)=1$, it follows that $0 \leq \Phi_{1}(t) \leq 1$.

In summary, the whole trajectory $\Phi(t)$ lives in the gray section indicated in Fig. 1 . 


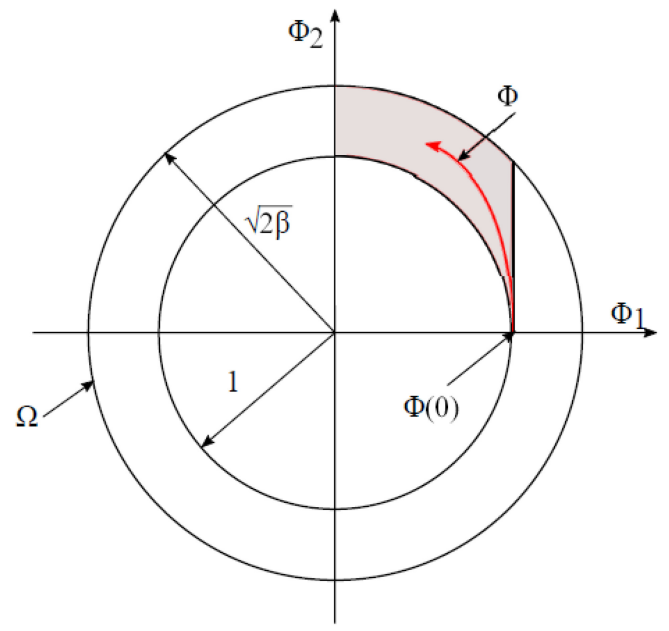

Figure 1: Behavior of the trajectory $\Phi$.

115

\section{(ii) Proof of boundedness of $\Phi(k)$}

Replacing (19) in (6)-(8) yields the dynamics

$$
\Phi(k+1)=\left[\begin{array}{cc}
1 & -T \mu \Delta(k) \Phi_{1}(k) \\
T \mu \Delta(k) \Phi_{1}(k) & 1-T \tilde{V}(k)
\end{array}\right] \Phi(k) .
$$

Hence, computing

$$
\begin{aligned}
|\Phi(k+1)|^{2} & =\Phi^{\top}(k)\left[\begin{array}{cc}
1 & T \mu \Delta(k) \Phi_{1}(k) \\
-T \mu \Delta(k) \Phi_{1}(k) & 1-T \tilde{V}(k)
\end{array}\right]\left[\begin{array}{cc}
1 & -T \mu \Delta(k) \Phi_{1}(k) \\
T \mu \Delta(k) \Phi_{1}(k) & 1-T \tilde{V}(k)
\end{array}\right] \Phi(k) \\
& =\Phi^{\top}(k)\left[\begin{array}{cc}
1 & 0 \\
0 & 1-2 T \tilde{V}(k)
\end{array}\right] \Phi(k)+T^{2} \Phi^{\top}(k)\left[\begin{array}{cc}
\mu^{2} \Delta^{2}(k) \Phi_{1}^{2}(k) & -\mu \Delta(k) \tilde{V}(k) \Phi_{1}(k) \\
-\mu \Delta(k) \tilde{V}(k) \Phi_{1}(k) & \mu^{2} \Delta^{2}(k) \Phi_{1}^{2}(k)+\tilde{V}^{2}(k)
\end{array}\right] \Phi(k) \\
& =|\Phi(k)|^{2}-2 T \tilde{V}(k) \Phi_{2}^{2}(k)+T^{2} \Phi^{\top}(k)\left[\begin{array}{cc}
\mu^{2} \Delta^{2}(k) \Phi_{1}^{2}(k) & -\mu \Delta(k) \tilde{V}(k) \Phi_{1}(k) \\
-\mu \Delta(k) \tilde{V}(k) \Phi_{1}(k) & \mu^{2} \Delta^{2}(k) \Phi_{1}^{2}(k)+\tilde{V}^{2}(k)
\end{array}\right] \Phi(k) .
\end{aligned}
$$

Invoking the assumption that $T^{2} \approx 0$ we obtain

$$
\tilde{V}(k+1)=\tilde{V}(k)-T \tilde{V}(k) \Phi_{2}^{2}(k)
$$

from which we conclude the invariance of the set (24).

Following verbatim the reasoning carried out in CT we conclude that the whole trajectory $\left(\Phi_{1}(k), \Phi_{2}(k)\right)$ is inside the disk described by the set $\Omega$, that is,

$$
\tilde{V}(k) \leq 0, \forall k \in \mathbb{Z}_{\geq 0}
$$

Replacing the bound above in 28 we have that $\tilde{V}(k)$ is non-decreasing, hence

$$
\tilde{V}(k) \geq \tilde{V}(0)=\frac{1}{2}-\beta .
$$

Combining the bounds $(29)$ and $(30)$ we conclude that

$$
1 \leq \Phi_{1}^{2}(k)+\Phi_{2}^{2}(k) \leq 2 \beta .
$$


As done in CT the bounds above can be further sharpened as follows. From 27) we have that

$$
\Phi_{2}(k+1)=[1-T \tilde{V}(k)] \Phi_{2}(k)+T \mu \Delta(k) \Phi_{1}^{2}(k) .
$$

From $\mu>0, \Delta(k) \geq 0$ and 25 it follows that $\Phi_{2}(k)$ is non-decreasing. Moreover, recalling the initial condition $\Phi_{2}(0)=0$, it follows that $\Phi_{2}(k) \geq 0$ for all $k \in \mathbb{Z}_{\geq 0}$.

Now, from 27] we also have that

$$
\Phi_{1}(k+1)=\left[1-T \mu \Delta(k) \Phi_{2}(k)\right] \Phi_{1}(k) .
$$

Since, the term in brackets belongs to the interval $[0,1)$ under a proper-sufficiently small-choice of $T$, we have that the sequence $\Phi_{1}(k)$ is non-increasing and, recalling that $\Phi_{1}(0)=1$, it follows that $0 \leq \Phi_{1}(k) \leq 1$.

In summary, the whole trajectory $\Phi(k)$ lives in the gray section indicated in Fig. 1

(iii) Proof of PE of $\Phi_{2}$

The assumption that $\Delta(t)$ in IE implies that there exists a $t_{0} \in\left(0, t_{c}\right]$ such that $\Delta\left(t_{0}\right)>0$, which in turn implies that there exists a $t_{\rho}>0$ such that $\rho:=\Phi_{2}\left(t_{\rho}\right)>0$. Since we proved above that $\Phi_{2}(t)$ is non-decreasing we have that

$$
\Phi_{2}(t) \geq \rho>0, \forall t \geq t_{\rho}
$$

Consequently,

$$
\liminf _{t \rightarrow \infty} \Phi_{2}(t)>0
$$

and $\Phi_{2}(t)$ is PE.7

Exaclty the same arguments can be used in DT to prove that

$$
\liminf _{k \rightarrow \infty} \Phi_{2}(k)>0
$$

hence $\Phi_{2}(k)$ is PE.

(iii) Boundedness of $z$ and $\xi$

From [15] — and the equivalent relation in CT [5] — we have that

$$
x=\left[\begin{array}{l}
\theta \\
z
\end{array}\right]=\xi+\Phi \theta .
$$

Since we proved that $\Phi$ is bounded, to establish boundedness of $\xi$ it suffices to prove that $z$ is bounded. Towards this end, we replace $(18)$ or $(19)$ in the $z$ dynamics of $(6 a)$ to get

$$
\begin{aligned}
\dot{z}(t) & =-\tilde{V}(t) z(t)+\mu \Phi_{1}(t) \mathcal{Y}(t)+u_{4}(t) \\
& =-\mu z(t)+\mu \Phi_{1}(t) \mathcal{Y}(t),
\end{aligned}
$$

\footnotetext{
${ }^{7}$ It is well-known that a scalar signal (with a bounded derivative) that does not converge to zero is PE.
} 
in $\mathrm{CT}$ and

$$
\begin{aligned}
z(k) & =[1-T \tilde{V}(k)] z(k)+T \mu \Phi_{1}(k) \mathcal{Y}(k)+u_{4}(k) \\
& =(1-b) z(k)+T \mu \Phi_{1}(k) \mathcal{Y}(k),
\end{aligned}
$$

in DT. In both cases, we are dealing with asymptotically stable LTI filters with bounded input, completing the proof.

\section{Discussion}

The following remarks are in order.

- The main message of Proposition 4 is that it is possible to estimate the parameters of a classical vector LRE (1) even when the regressor $\Omega$ is not PE - the convergence of the new parameter estimator being global and exponential 8

- The choice of the signals $u(k)$ given in 19 is motivated by the CT dynamics 222. Indeed, the DT dynamics of $\Phi(k)$ given in (27) is the Euler approximation of 222 . It is well-known 22] that the Euler approximation is a numerical integration method of order one whose global approximation error is $\mathcal{O}\left(T^{2}\right) \unlhd^{9}$ This explains our need to impose the assumption (21) in our stability analysis. It should be underscored that if we remove this assumption the result is not valid anymore. However, notice that $T$ is a designer chosen constant. See also the discussion pertaining this issue in Section 6 .

140

- Although it is possible to consider other (higher order) discretization methods of the $\Phi(t)$ dynamics $(22)$, the resulting discretized dynamics cannot be matched with the $A(k)$ matrix given in $(8)$ due to the fact that - as seen in (9) - it is necessary to have the term $A_{11}(k)=1$. A condition that stymies the selection of a more precise discretization method.

- Another alternative to remove the undesirable assumption (21) is to directly pose a regulation problem for the DT system identified in Proposition 2 , that is

$$
\Phi(k+1)=\left[\begin{array}{cc}
1 & u_{1}(k) \\
u_{2}(k) \Delta(k) & u_{3}(k)
\end{array}\right] \Phi(k) .
$$

The task is to select the signals $u_{i}(k), i=1,2,3$, that insure boundedness of all signals and that $\Phi_{2}(k)$ is PE. Unfortunately, this a highly complicated nonlinear control problem with non-standard regulation objectives.

- In [5] the proof of boundedness of the signal $z(t)$ is quite involved and requires the addition of an unverifiable absolute integrability assumption [5, Equation (14)]. This is due to the fact that the new free signal $u_{4}(t)$ in the

\footnotetext{
${ }^{8}$ Additional properties of the DREM estimator, like element-by-element monotonicity of the parameter errors, may be found in [17.

${ }^{9} f(t, T)$ is $\mathcal{O}\left(T^{2}\right)$, refered in the literature as "big o of $T^{2}$ ", if and only if $|f(t, T)| \leq C T^{2}$ with $C$ a constant independent of $t$ and $T$.
} 
vector $b(t)$ in $(8)$, was not included in [5]. It is clear that the addition of this signal does not affect the main result, and trivializes the proof of boundedness of $z(t)$.

\section{Application to Identification of CT Systems in Unexcited Conditions}

To illustrate the result of Proposition 4, we consider in this section the problem of parameter estimation of an CT LTI system and choose, as an example, the system:

$$
y_{p}(t)=\frac{B(p)}{A(p)}\left[u_{p}\right](t)=\frac{b_{1} p+b_{0}}{p^{2}+a_{1} p+a_{0}}\left[u_{p}\right](t),
$$

where $u_{p}(t) \in \mathbb{R}$ and $y_{p}(t) \in \mathbb{R}$ are the control and output signals, respectively. Following the standard procedure [21, Subsection 2.2] we derive the LRE (1) for the system (31) as follows

$$
\mathbf{Y}(t):=y_{p}(t), \Omega(t):=\left[\begin{array}{c}
\frac{F(p) B(p)}{A(p)} \\
F(p)
\end{array}\right]\left[u_{p}\right](t), F(p):=\frac{1}{\lambda(p)}\left[\begin{array}{c}
1 \\
p \\
\vdots \\
p^{n-1}
\end{array}\right], \theta:=\left[\begin{array}{c}
\lambda_{0}-a_{0} \\
\vdots \\
\lambda_{n-1}-a_{n-1} \\
b_{0} \\
\vdots \\
b_{n-1}
\end{array}\right]
$$

with $\lambda(p)=\sum_{i=0}^{n} \lambda_{i} p^{i}, \lambda_{n}=1$, an arbitrary Hurwitz polynomial.

We consider the following simulation scenarios.

S1 Estimation of the vector $\theta$ with the standard gradient estimator

$$
\dot{\hat{\theta}}(t)=\Gamma \Omega(t)\left[\mathbf{Y}(t)-\Omega^{\top}(t) \hat{\theta}(t)\right], \Gamma>0
$$

using the original vector LRE (1).

S2 Estimation of the parameters $\theta_{i}$ using the scalar regression form 5 obtained via the KRE and DREM of Proposition 1, that is

$$
\dot{\hat{\theta}}_{i}(t)=\gamma_{i} \Delta(t)\left[\mathcal{Y}_{i}(t)-\Delta(t) \hat{\theta}_{i}(t)\right], \gamma_{i}>0
$$

S3 Estimation of the parameters $\theta_{i}$ using the new scalar regression form 10 obtained via the LRE generator of Proposition 4, that is

$$
\dot{\hat{\theta}}_{i}(t)=\gamma_{i} \Phi_{2}(t)\left(Y_{i}(t)-\Phi_{2}(t) \hat{\theta}_{i}(t)\right), \gamma_{i}>0
$$

S4 Simulation of the three estimators above for a sufficiently rich input signal

$$
u_{p a}(t)=\sin (2 \pi t)+\cos (3 t)
$$

and for an input signal that is not sufficiently rich, but generates a regressor $\Omega$ which is IE, namely

$$
u_{p b}(t)=e^{-2 t}+e^{-1.5 t} .
$$




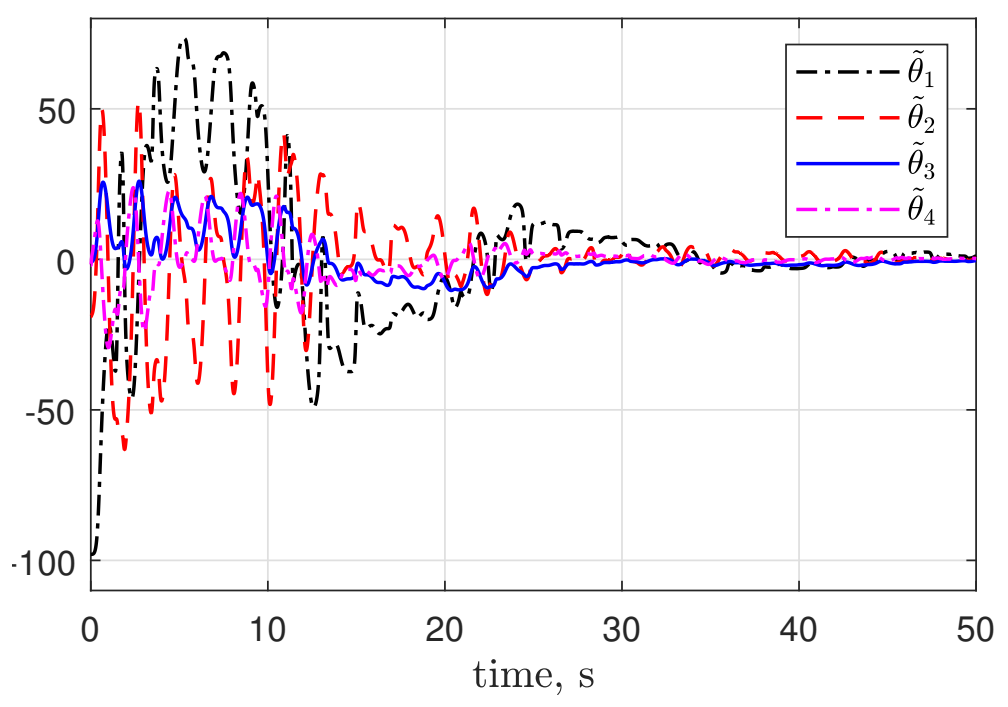

Figure 2: $\tilde{\theta}(t)$ with gradient algorithm 33 and $u_{p a}(t)$

The following remarks concerning the theoretical results are in order

- For the sufficiently rich signal (36) the three estimators yield consistent estimates.

- For the not sufficiently rich signal (37) the first and second estimators will not generate consistent estimates. For the first estimator this follows from the fact that, as shown in 21, Theorems 2.7.2 and 2.7.3] sufficient richness of the plants input signal is equivalent to PE of the regressor $\Omega(t)$. Regarding the DREM estimator with the regressor $\Delta(t)$, it was shown in [1, Proposition 2] that DREM alone cannot relax the PE condition in the system identification problem. Hence, sufficient richness is necessary for parameter convergence.

- On the other hand, the result of Proposition 4 ensures that DREM with the new LRE will ensure convergence even for the input signal (37).

The simulations were carried out for the system studied in [1, Section 5], that is $\left(a_{0}, a_{1}, b_{0}, b_{1}\right)=(2,1,1,2)$ and we choose $\lambda_{1}=20$ and $\lambda_{0}=100$. This yields $\theta=\left[\begin{array}{llll}98 & 19 & 1 & 2\end{array}\right]$.

For all estimators we set $\hat{\theta}_{i}(0)=0$. The gain matrix for the first algorithm $(33)$ is

$$
\Gamma=100 \operatorname{diag}(100,50,30,10) .
$$

For the estimators (34) and(35) we choose $\gamma_{i}=1, i=1, \ldots, 4$. The parameters of the KRE (2) are $g=100, \lambda=30$. For the new LRE we choose $\beta=0.51$.

The simulation results, which corroborate the claims above, are shown in Figs. 217. Notice, in particular, that for the input signal (37) only DREM with the new LRE ensures convergence.

To test the robustness of the various estimators bounded noise was added to the output signal, as shown in Fig. 8 for the case of the input $u_{p b}(t)$.

As expected, in this case none of the estimators ensures that the error $\tilde{\theta}(t)$ converges to zero, as shown in Figs. 9 11. However, notice that the gradient algorithm (33) actually diverges. On the other hand, while the steady state 


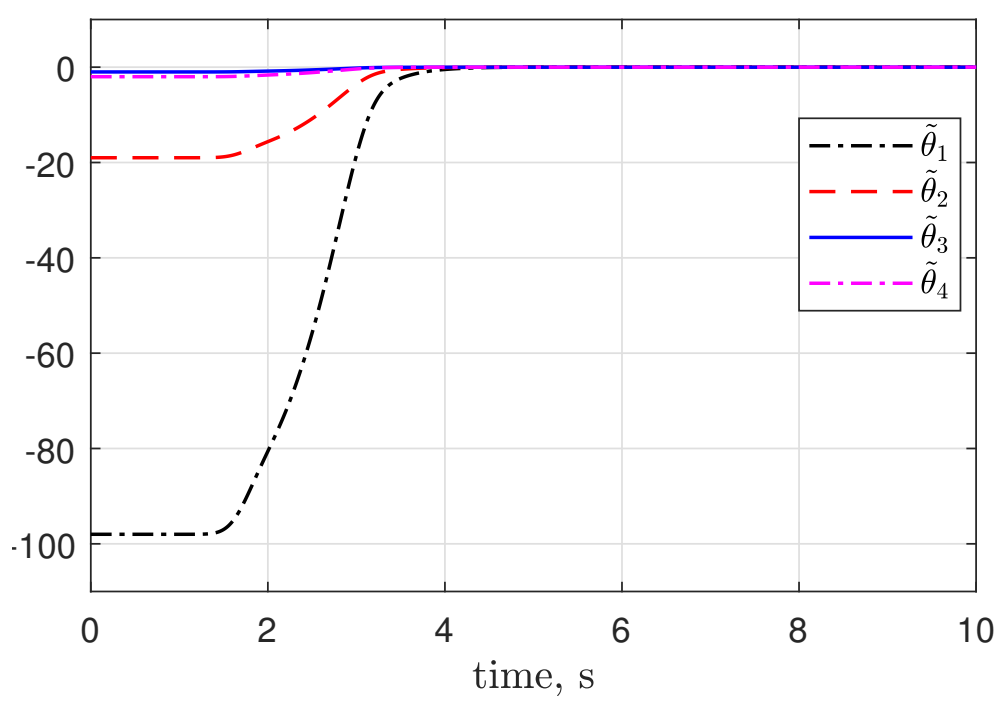

Figure 3: $\tilde{\theta}(t)$ with DREM procedure, estimator 34 and $u_{p a}(t)$

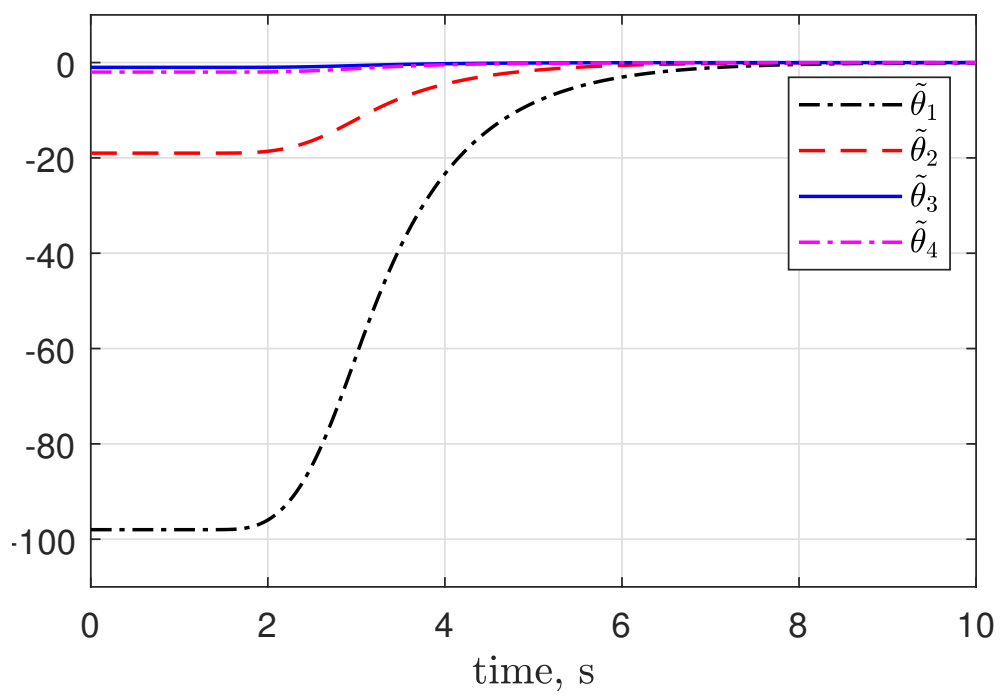

Figure 4: $\tilde{\theta}(t)$ with DREM procedure and new LRE, estimator 35 and $u_{p a}(t)$

error of the estimator with DREM (34) is quite large, the one of (35) with the new LRE, is negligible - illustrating the robustness to additive noise of the the new scheme.

\section{Simulations of the Discrete-time LRE Generator}

In this section, we present comparative simulations of the DT estimation of a scalar parameter $\theta \in \mathbb{R}$ using the standard gradient descent adaptation with the original and the new regressor, that is,

$$
\begin{aligned}
\hat{\theta}_{\text {ori }}(k+1) & =\hat{\theta}_{\text {ori }}(k)+\frac{\gamma \Delta(k)}{1+\gamma \Delta^{2}(k)}\left[\mathcal{Y}(k)-\Delta(k) \hat{\theta}_{\text {ori }}(k)\right] \\
\hat{\theta}_{\text {new }}(k+1) & =\hat{\theta}_{\text {new }}(k)+\frac{\gamma \Phi_{2}(k)}{1+\gamma \Phi_{2}^{2}(k)}\left[Y(k)-\Phi_{2}(k) \hat{\theta}_{\text {new }}(k)\right],
\end{aligned}
$$




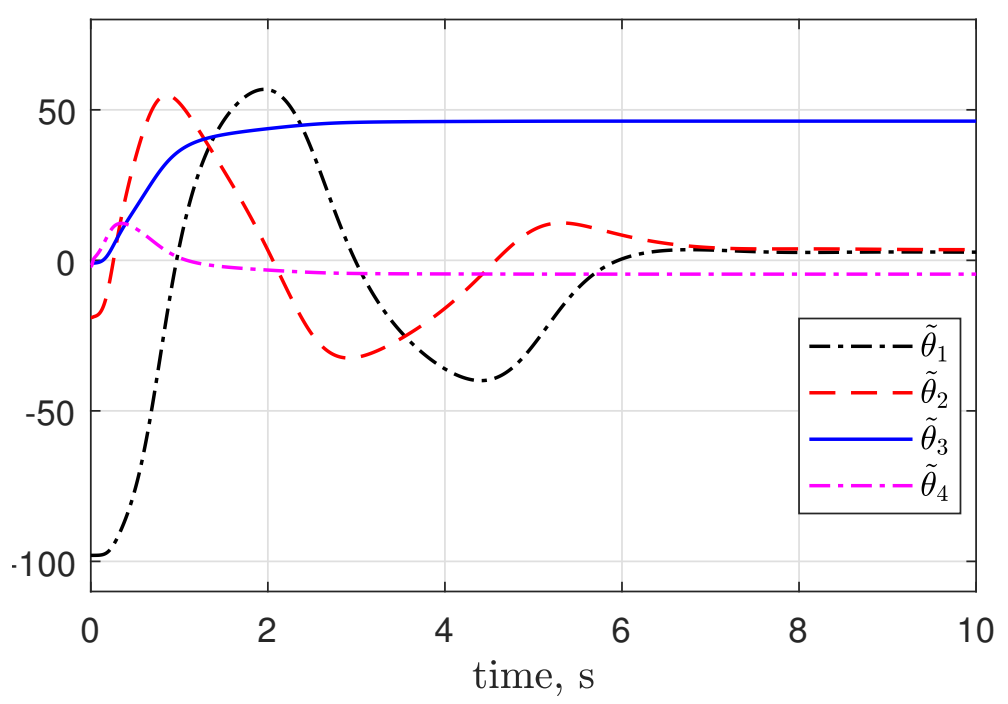

Figure 5: $\tilde{\theta}(t)$ with gradient algorithm 33 and $u_{p b}(t)$

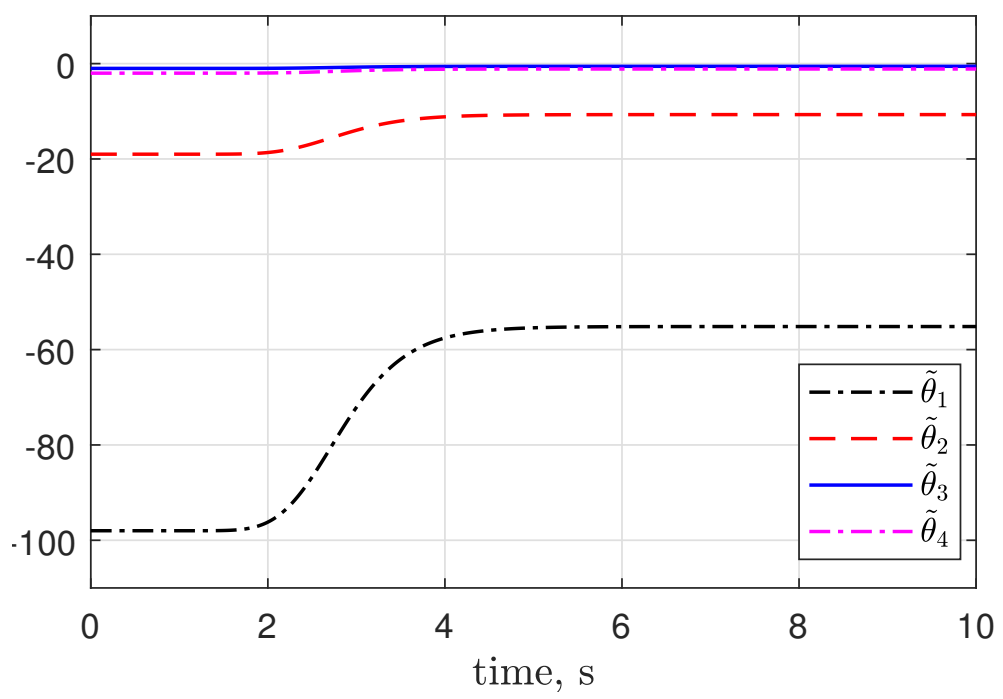

Figure 6: $\tilde{\theta}(t)$ with DREM procedure, estimator 34 and $u_{p b}(t)$

with $\gamma>0$ for three different signals $\Delta(k)$, namely:

$$
\begin{aligned}
& \Delta_{a}(k)=e^{-3 t} \\
& \Delta_{b}(k)=\left\{\begin{array}{lr}
1 & t \in[0,0.2] \\
0 & t>0.2
\end{array}\right. \\
& \Delta_{c}(k)=\frac{1}{7+t} .
\end{aligned}
$$

with $t=k T$.

Clearly, the three signals are not PE and belong to $\mathcal{L}_{2}$. Hence, according with Proposition 3 the estimator (38) will not converge. On the other hand, since they are IE, the estimator (39) should guarantee convergence for small values of $T$.

\footnotetext{
In the first simulations we consider the unknown parameter $\theta=5$ and select $\beta=\frac{3}{4}, \mu=0.4, b=0.1$ and $\gamma=0.2$.
} 


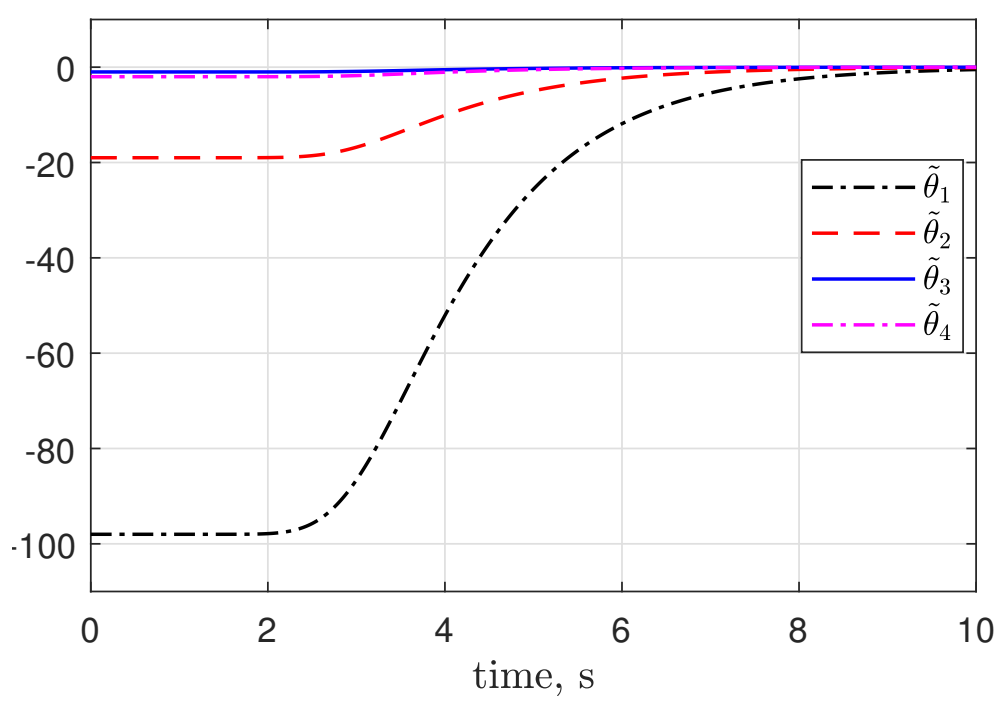

Figure 7: $\tilde{\theta}(t)$ with DREM procedure and new LRE, estimator 35 and $u_{p b}(t)$

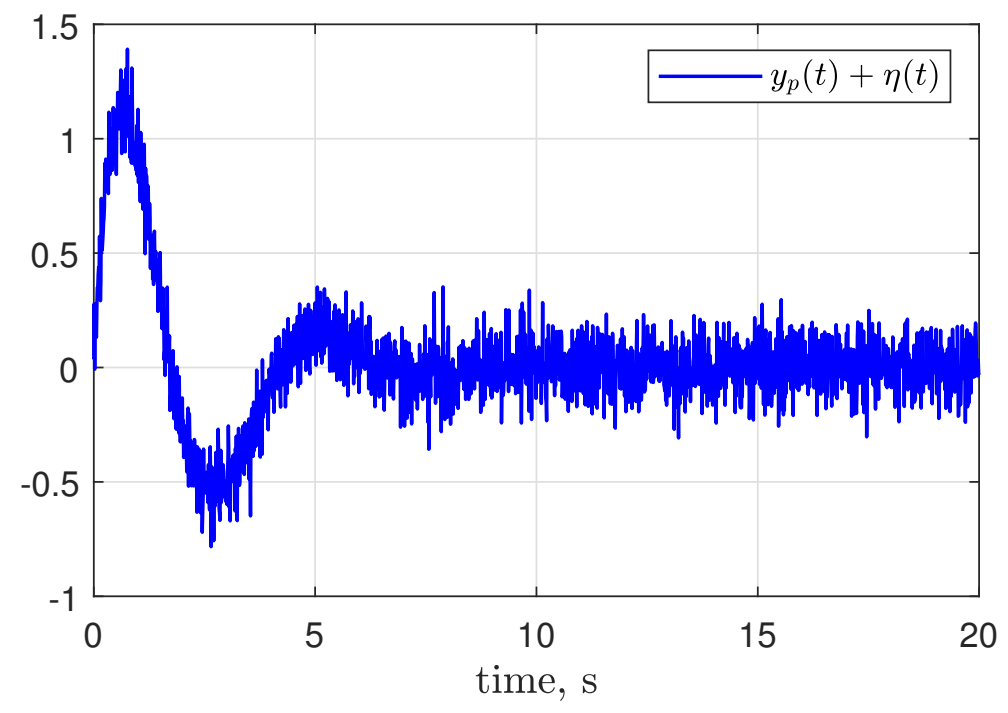

Figure 8: $y_{p}(t)$ with additive noise $\eta(t)$

The initial conditions of the estimators are set as $\hat{\theta}_{\text {ori }}(0)=0$ and $\hat{\theta}_{\text {new }}(0)=0$. In the light of the key assumption 21. we also check the effect of the size of the constant $T$, carrying out simulations using the values $T=0.01, T=0.1$ and $T=1.5$.

The results of these simulations are given in Figs. 12, 14, that confirm the predictions of the theoretical analysis. We also notice that taking a large value for $T$ does not affect the steady-state performance, but it increases significantly the convergence time. The rationale for this behavior may be explained as follows. In the scenarios considered above the signals $\Delta(k)$ converge to zero, reducing the effect of the truncation error.

The situation is different if $\Delta(k)$ does not converge to zero, for instance if it is PE. In that case, it is expected that the performance is degraded with increasing values of $T$. To validate this conjecture we carried out a simulation with the PE signal $\Delta_{d}(k)=\cos \left(\frac{\pi}{4} k\right)$. In this case the estimator (38) always converges. However, (39) will ensure parameter convergence only for small values of $T$. This is corroborated with the plots of Fig. 15 that show how the 


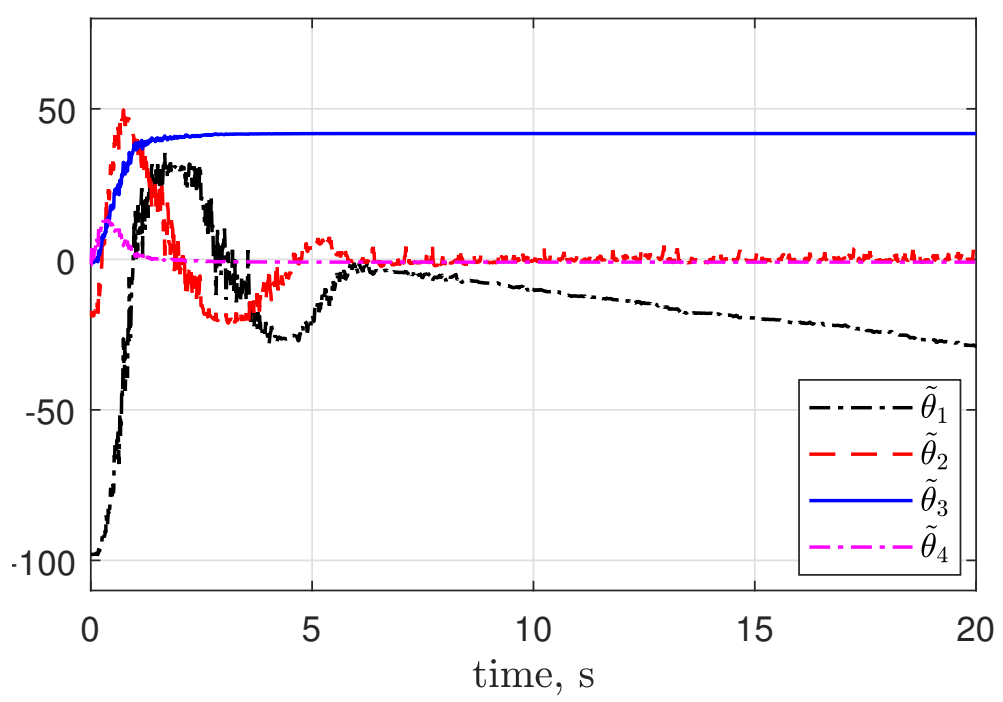

Figure 9: $\tilde{\theta}(t)$ (under the influence of $\eta(t)$ ) with gradient algorithm 33 and $u_{p b}(t)$

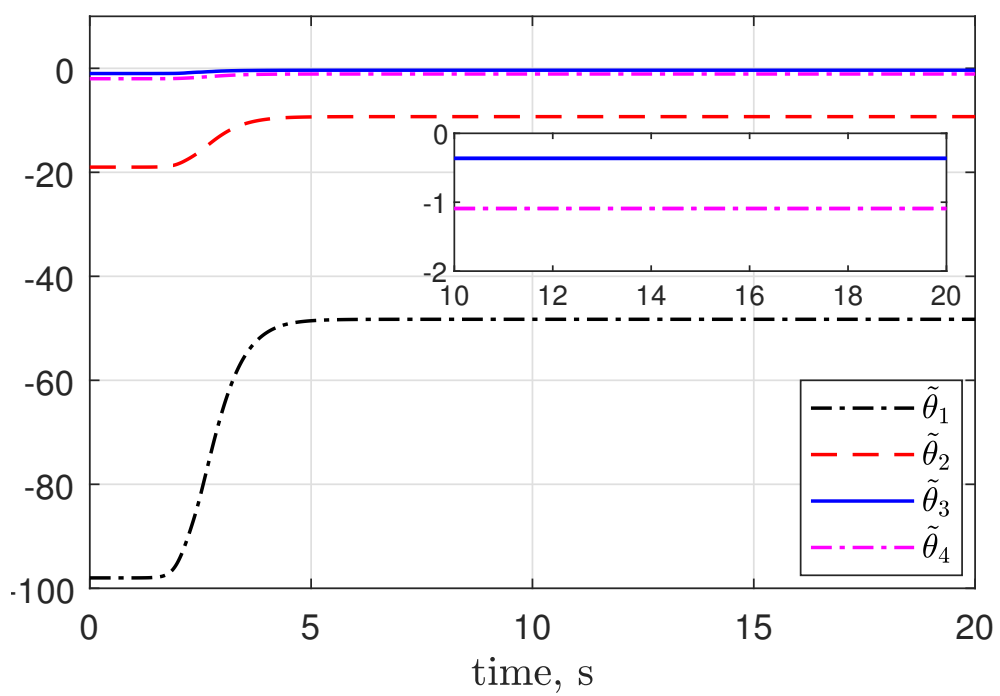

Figure 10: $\tilde{\theta}(t)$ (under the influence of $\eta(t)$ ) with DREM procedure, estimator 34 and $u_{p b}(t)$

performance of the estimator (39) degrades with increasing $T$. Moreover, from the phase portrait we notice that for $T=1.5$ the signal $\Phi(k)$ does not live in the gray section indicated in Fig. 1, violating the predictions of the theory because assumption (21) is not valid anymore. It is important to underscore that the main motivation for the introduction of the LRE generator is for the case when the original regressor is not PE, therefore the scenario considered in these simulations will not be encountered in practice.

\section{Conclusions and Future Research}

We have proposed new CT and DT estimators for the LRE (1) that ensure global exponential convergence under the weak assumption that the regressor $\Omega$ is IE. To the best of our knowledge, this is the first time that such a result 


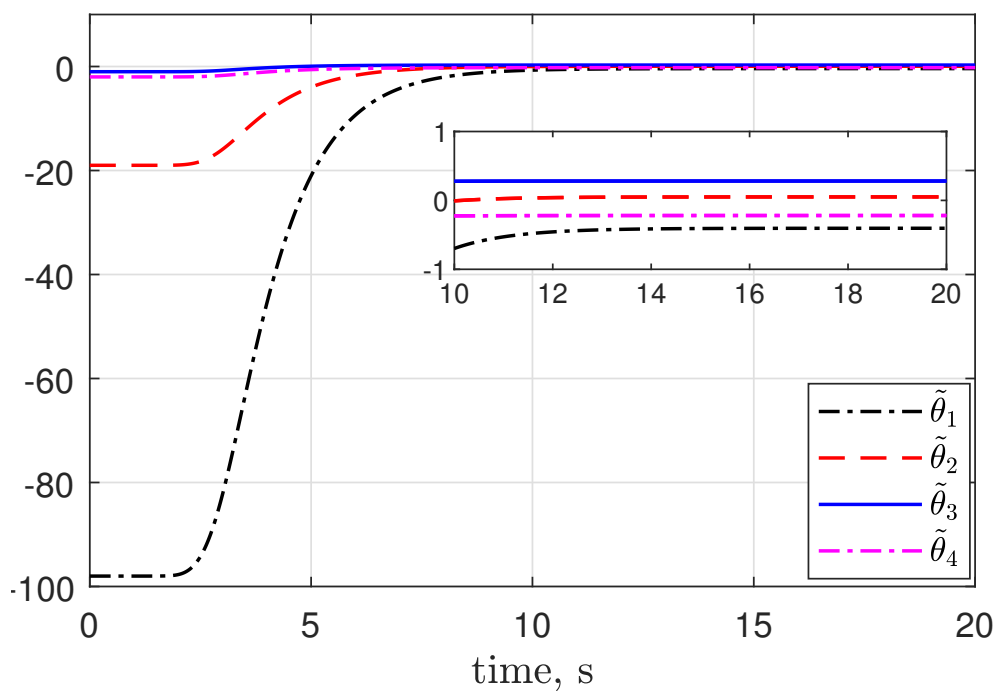

Figure 11: $\tilde{\theta}(t)$ (under the influence of $\eta(t)$ ) with DREM procedure and new filter, estimator 35 and $u_{p b}(t)$

is established for an estimator that does not rely on off-line data memory manipulation ${ }^{10}$ It should be noticed, however, that the estimator is quite computationally demanding, requiring the introduction of various filters and algebraic operations. Moreover, experience has shown that tuning of the various gains of the algorithm - that is now done via trail-and-error - is far from obvious.

Our current efforts are directed towards the relaxation of the assumption (21) for the DT estimator and to further study the effect of noise in the estimators performance - in particular carrying out a theoretical analysis.

\section{References}

[1] S. Aranovskiy, A. Belov, R. Ortega, N. Barabanov and A, Bobtsov, Parameter identification of linear timeinvariant systems using dynamic regressor extension and mixing. International Journal of Adaptive Control and Signal Processing, vol. 33, no. 6, pp. 1016-1030, 2019.

[2] S. Aranovskiy, A. Bobtsov, R. Ortega and A. Pyrkin, Performance enhancement of parameter estimators via dynamic regressor extension and mixing, IEEE Trans. on Automatic Control, vol. 62, pp. 3546-3550, 2017. (See also arXiv:1509.02763 for an extended version.)

[3] S. Aranovskiy, R. Ushirobira, M. Korotina and A. Vedyakov, On preserving-excitation properties of a dynamic regressor extension scheme, IEEE Trans. on Automatic Control, (submitted, see also https://halcentralesupelec.archives-ouvertes.fr/hal-03245139/document), 2022.

[4] A. Belov, R. Ortega and A. Bobtsov, Guaranteed performance adaptive identification scheme of discrete-

\footnotetext{
${ }^{10}$ As mentioned in Section 1 the concurrent [6] and composite learning [20] estimators involve an off-line operation of data monitoring and stacking. See also 13 where it is shown that allowing off-line calculations it is possible to ensure finite convergence time with an IE assumption.
} 

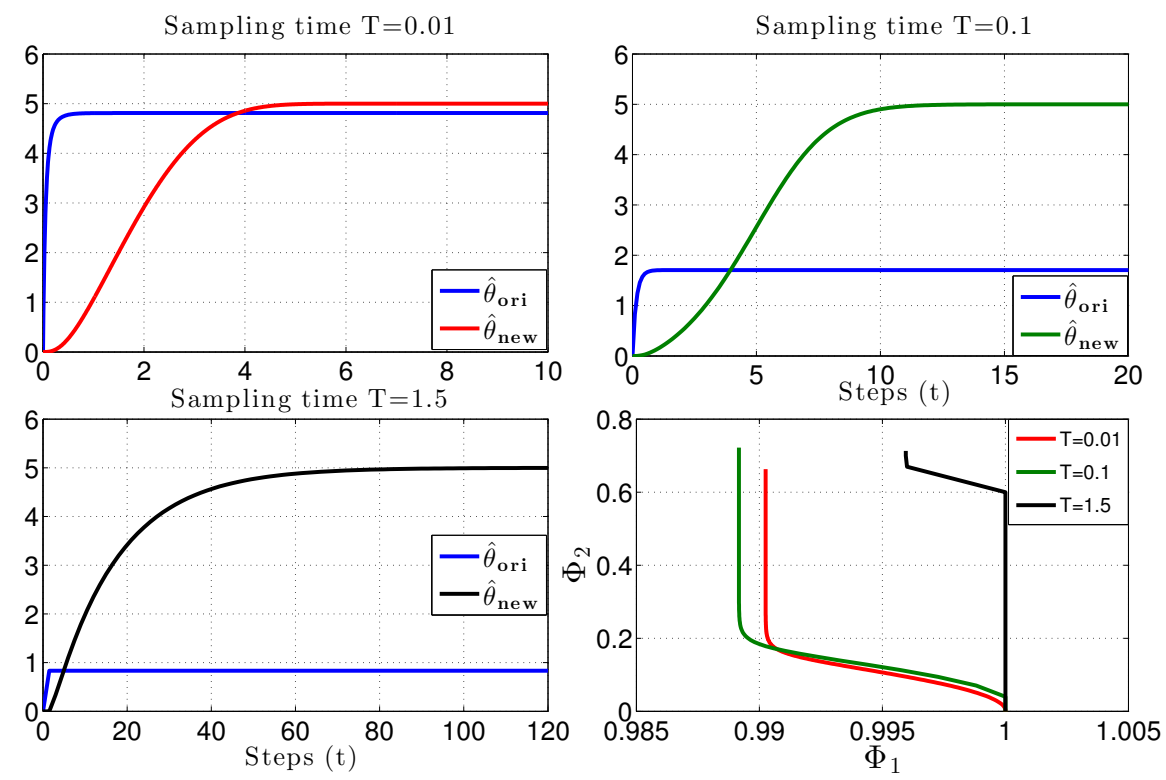

Figure 12: Estimates using the original and new LRE and the phase portrait of $\Phi(k)$ with the signal $\Delta_{a}(k)$
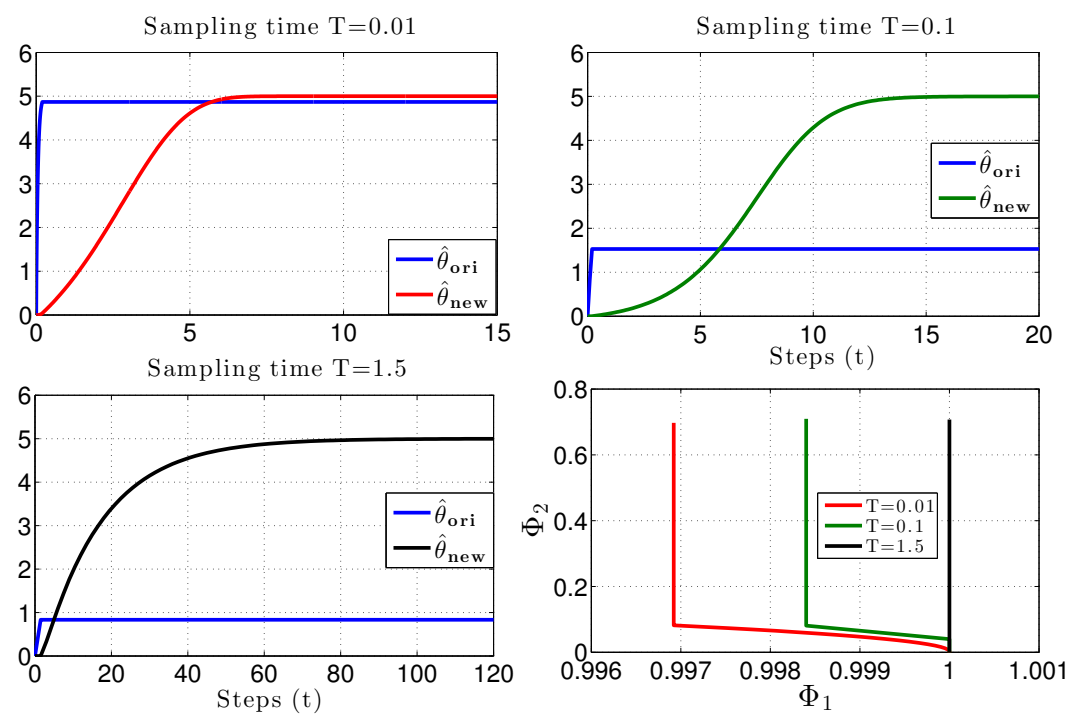

Figure 13: Estimates using the original and new LRE and the phase portrait of $\Phi(k)$ with the signal $\Delta_{b}(k)$ 

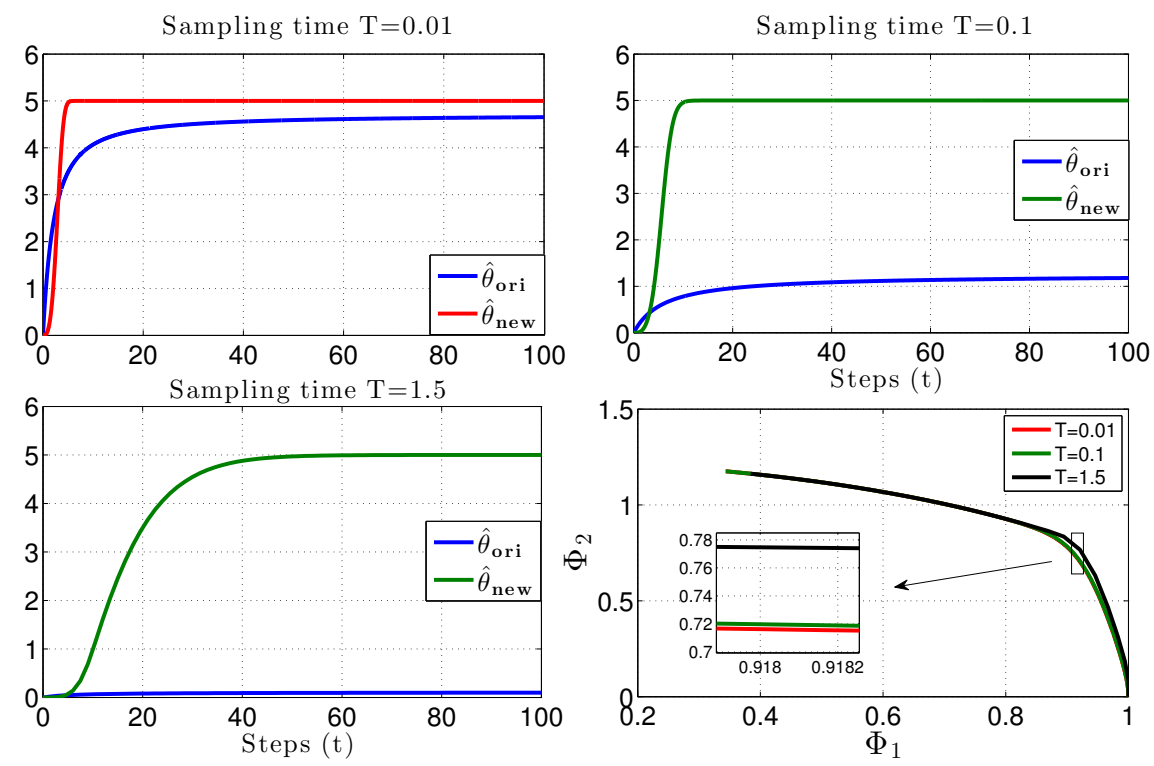

Figure 14: Estimates using the original and new LRE and the phase portrait of $\Phi(k)$ with the signal $\Delta_{c}(k)$
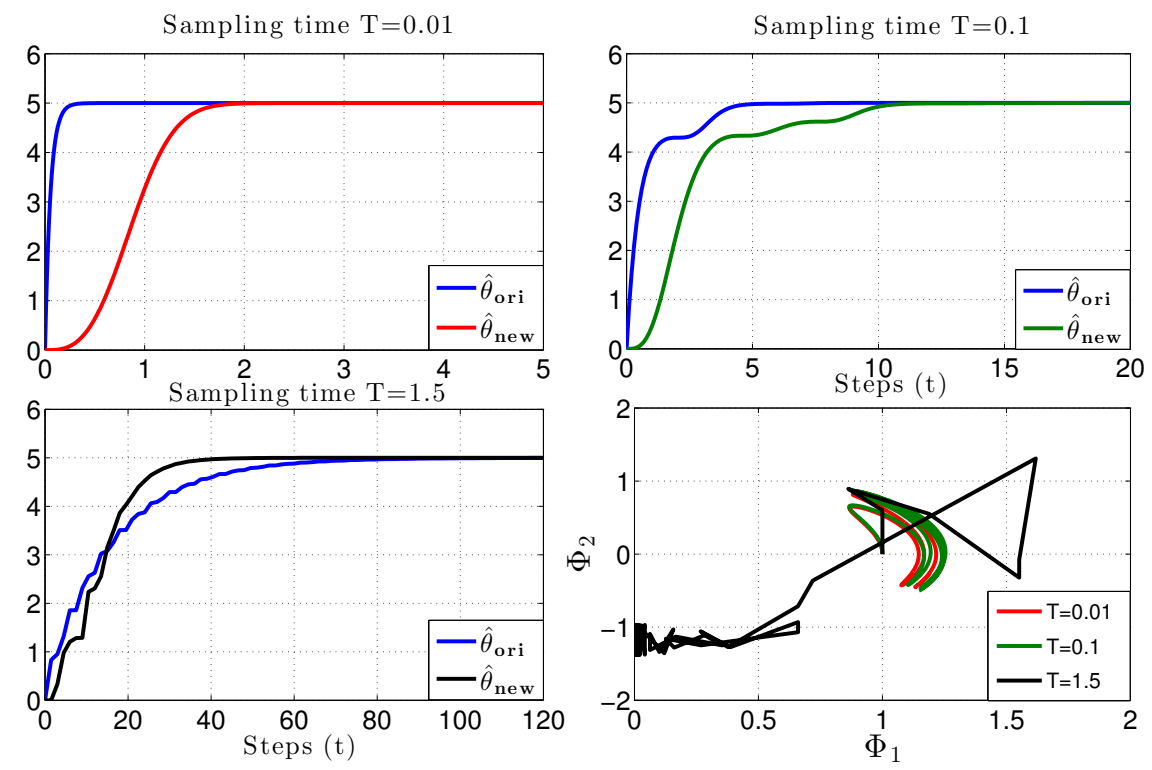

Figure 15: Estimates using the original and new LRE and the phase portrait of $\Phi(k)$ with the signal $\Delta_{d}(k)$ 
time systems using dynamic regressor extension and mixing, 18th IFAC Symposium on System Identification, (SYSID 2018), Stockholm, Sweden, July 9-11, 2018.

[5] A. Bobtsov, B. Yi, R. Ortega and A. Astolfi, Generation of new exciting regressors for consistent on-line

[18] R. Ortega, V. Gromov, E. Nuño, A. Pyrkin and J. G. Romero, Parameter estimation of nonlinearly parameterized regressions: application to system identification and adaptive control, Automatica, vol.. 127, 109544, May 2021. 


\begin{tabular}{l|r}
\hline \hline CT & Continuous-time \\
DREM & Dynamic regressor extension and mixing \\
DT & Discrete-time \\
IE & Interval excitation \\
KRE & Kreisselmeier's regressor extension \\
LRE & Linear regressor equation \\
LTI & Linear time-invariant \\
PE & Persistent excitation \\
\hline \hline
\end{tabular}

Table 1: List of Acronyms

[22] J. Stoer and R. Bulirsch, Introduction to Numerical Analysis, Springer-Verlag, NY, 1983.

[23] B. Yi, R. Ortega, D. Wu and W. Zhang, Orbital stabilization of nonlinear systems via Mexican sombrero energy pumping-and-damping injection. Automatica, vol. 112, 108-861, 2020. 